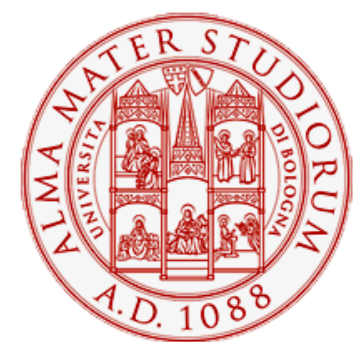

Alma Mater Studiorum - Università di Bologna DEPARTMENT OF ECONOMICS

\title{
On the Optimal Number of Firms in the Commons: Cournot vs Bertrand
}

Davide Dragone

Luca Lambertini

Arsen Palestini

Alessandro Tampieri

Quaderni - Working Paper DSE $N^{\circ} 856$

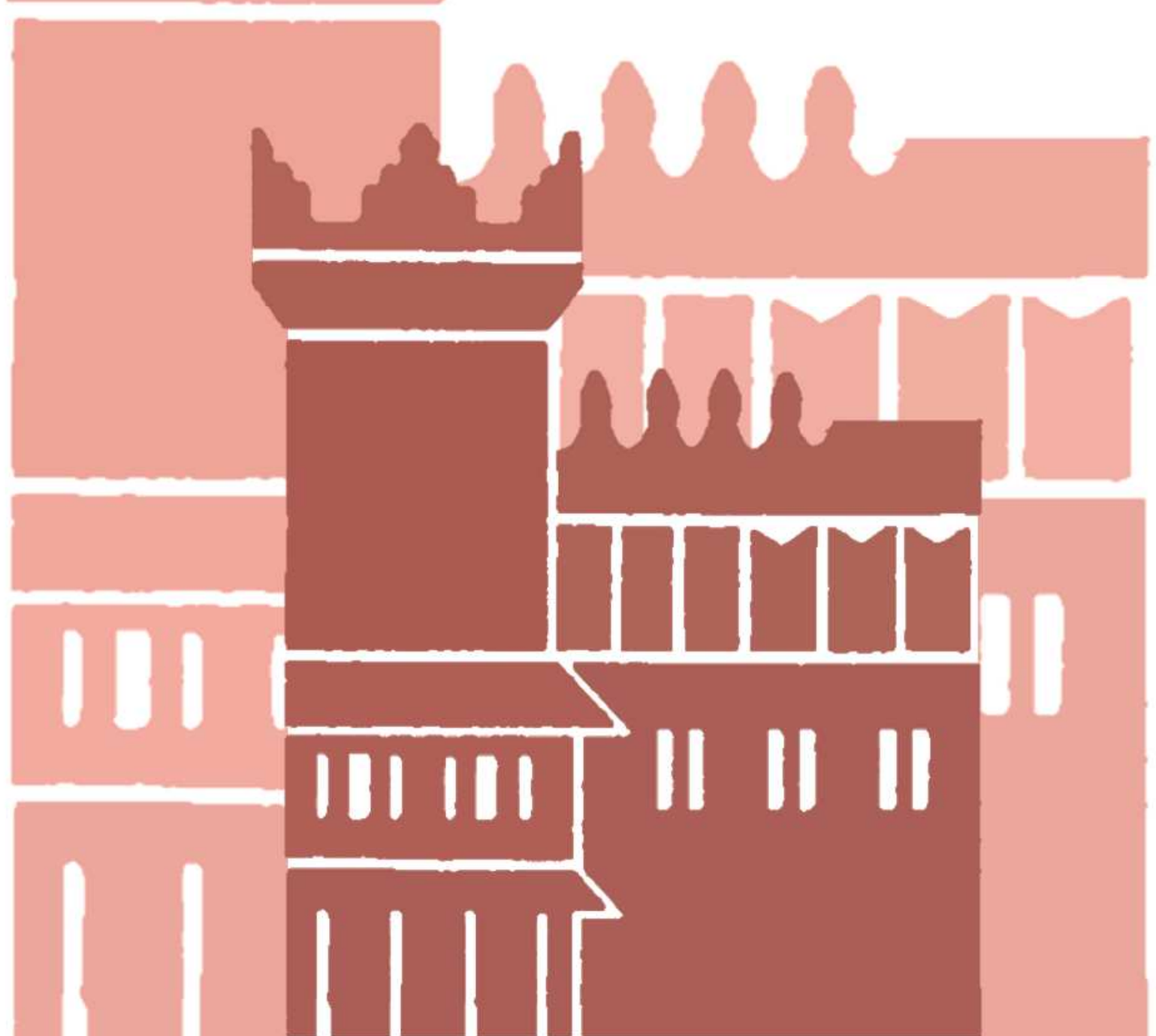




\title{
On the Optimal Number of Firms in the Commons: Cournot vs Bertrand*
}

\author{
Davide Dragone\# ${ }^{\#}$, Luca Lambertini\#, \\ Arsen Palestini ${ }^{\S}$ and Alessandro Tampieri\# \\ \# Department of Economics, University of Bologna \\ Strada Maggiore 45, 40125 Bologna, Italy \\ davide.dragone@unibo.it; luca.lambertini@unibo.it; alessandro.tampieri@unibo.it \\ $\S$ MEMOTEF, University of Rome La Sapienza \\ via del Castro Laurenziano 9, I-00161 Roma, Italy \\ arsen.palestini@uniroma1.it
}

December 11, 2012

\begin{abstract}
We revisit the debate on the optimal number o firms in the commons in a differential oligopoly game in which firms are either quantityor price-setting agents. Production exploits a natural resource and involves a negative externality. We calculate the number of firms maximising industry profits, finding that it is larger in the Cournot case. While industry structure is always ineffcient under Bertrand behaviour, it may or may not be so under Cournot behaviour, depending on parameter values. The comparison of private industry optima reveals that the Cournot steady state welfare level exceeds the corresponding Bertrand magnitude if the weight of the stock of pollution is large enough.
\end{abstract}

Keywords: natural resources, oligopoly, pollution, tragedy of commons

JEL Codes: C73, L13, Q20, Q51

*We thank the audience at IFAC CAO'12 (Rimini) for precious comments. The usual disclaimer applies. 


\section{Introduction}

The usual approach to the economics of the environment treats externalities and the extraction of natural resources separately, and in the latter case compares open access (or equivalently, perfect competition) against monopoly. ${ }^{1}$ Here we propose a unified approach to the two aspects of the industrial exploitation of the environmen, using a homogeneous good oligopoly in which firms may set either quantities or prices to maximise profits, and their productive activities require the use of a renewable resource and emit pollutants. Each of these two aspects has indeed received attention in the literature, either in static or in dynamic oligopoly models, ${ }^{2}$ but, to the best of our knowledge, the joint analysis of resource extraction and pollution has not. Our analysis will abstract from the possibility of regulating firms' interaction via Pigouvian taxation/subsidization ${ }^{3}$ and/or pollution rights, ${ }^{4}$ to focus on the issue of the optimal number of firms in the commons. This problem lies at the intersection between the well known discussion about the tragedy of the commons (Gordon, 1954; Hardin, 1968) and the standard approach to the entry process belonging to the theory of industrial organization (Novshek, 1980; Suzumura and Kiyono, 1987; Mankiw and Whinston, 1990). The backbone of this discussion is the fact that, while in absence of any external effects increasing competition (and therefore industry output) increases welfare, if industrial activities exploit natural resources and/or imply the emission of pollutants then the socially optimal degree of concentration of such an industry is determined by the balance between the price effect and the environmental one (Cornes and Sandler, 1983; Cornes, Mason and Sandler, 1986; Karp, 1992; Mason and Polasky, 1997). We revisit this issue in a differential game in which we assess the privately optimal structure (maximising industry profits) against the socially optimal industry structure (maximising social welfare), given the profit-maximising behaviour of firms, under both Cournot and Bertrand competition. Industry structure is always socially inefficient under Bertrand behaviour, while it may or may not be so under Cournot behaviour, depending on the environment's efficiency in ab-

\footnotetext{
${ }^{1}$ See Dasgupta and Heal (1979), Kemp and Long (1980), Pearce and Turner (1989), Tisdell (2009) and Anderson (2010), inter alia.

${ }^{2}$ The related literature is too large to be cited entirely. See, inter alia, McMillan and Sinn (1984), Sinn (1984), Katsoulacos and Xepapadeas (1995) and Fujiwara (2009).

${ }^{3}$ See Karp and Livernois (1992) and Benchekroun and Long (1998, 2002).

${ }^{4}$ See von der Fehr (1993) and Sunnevåg (2003).
} 
sorbing pollution. Then, we establish that (i) the privately optimal structure in Cournot exceeds its counterpart in Bertrand, and consequently (ii) social welfare in the private optimum can be higher at the Cournot equilibrium, if the weight of pollution in the social welfare function is sufficiently high.

The basic model is laid out in section 2 . The non-cooperative equilibrium between profit-maximizing firms is outlined in section 3. Section 4 contains the analysis of the social planning equilibrium. The two regimes are comparatively assessed in section 5, while the mixed setting is investigated in section 6 . Concluding remarks are in section 7 .

\section{The setup}

Consider an oligopoly market over an infinite (continuous) time horizon, $t \in[0, \infty)$, in which $n \geq 2$ firms supply a homogeneous good, whose market demand function is

$$
p(t)=a-Q(t)
$$

at any time $t \in[0, \infty)$, with $a>0$ being a positive constant parameter measuring the reservation price and $Q(t)=\sum_{i=1}^{n} q_{i}(t)$ being the sum of all firms' output levels. Production takes place at decreasing returns to scale, with the same technology being common to all firms alike, so that firm $i$ 's instantaneous cost function is $C_{i}(t)=c q_{i}^{2}(t)$, with the constant $c>0 .^{5}$ The production of the final output goes along with a negative environmental externality whose instantaneous level is $\Theta(t)=\gamma S^{2}(t) / 2$, with $\gamma>0$ and $S(t)$ evolving over time according to the following dynamics:

$$
\frac{d S(t)}{d t} \equiv \dot{S}(t)=b Q(t)-\delta S(t)
$$

where $\delta>0$ is the decay rate of the stock and $b$ is a positive constant.

The Instantaneous consumer surplus $C S(t)$ is measured by the area below the demand function and above market price $p(t)$, minus the externality $\Theta(t)$ :

$$
C S(t)=\frac{Q^{2}(t)}{2}-\gamma \frac{S^{2}(t)}{2} .
$$

\footnotetext{
${ }^{5}$ We could have specified the cost function as $C_{i}(t)=z q_{i}(t)+c q_{i}^{2}(t)$, with $z>0$. This would be a useless complication, however, as one could as well think of the vertical intercept of the demand function as $a=\widehat{a}-z$, whereby the ensuing analysis would reproduce unmodified.
} 
It is worth noting that a contraction of output has ambiguous consequences over consumer surplus, due to the presence of a negative externality proportional to the output: on the one hand, shrinking output goes along with increasing market price, which is harmful; on the other hand, it entails reducing the environmental externality, which is desirable. The balance between these components will play a key role in the remainder of the analysis.

Additionally, the production of the final good makes use of a renewable natural resource whose stock $X(t)$ follows the state equation:

$$
\frac{d X(t)}{d t} \equiv \dot{X}(t)=\eta X(t)-v Q(t)
$$

with constants $\eta$ and $v$ strictly positive.

The instantaneous social welfare function, defined as the sum of industry profits and consumer surplus, writes as follows:

$$
\begin{gathered}
S W(t)=\sum_{i=1}^{n} \pi_{i}(t)+\frac{Q^{2}(t)}{2} \\
-\gamma \frac{S^{2}(t)}{2}+X(t)
\end{gathered}
$$

where $\pi_{i}(t)=[p(t)-c] q_{i}(t)$ is firm $i$ 's instantaneous profit function.

In the remainder of the paper, we investigate the non-cooperative unregulated open-loop game where firms compete either à la Cournot-Nash or à la Bertrand, alternatively, to maximise individual profits. In both cases, firm $i$ chooses its strategy (either quantity or price) to maximise the discounted individual profit flow:

$$
J_{i}(t)=\int_{0}^{\infty} \pi_{i}(t) e^{-\rho t} d t
$$

s.t. the state equations (2) and (4), and the initial conditions $S(0)=S_{0}$ and $X(0)=X_{0}$. Parameter $\rho>0$ represents the constant discount rate common to all firms in the industry.

\section{The Cournot-Nash game}

Here we characterise the open-loop equilibrium of the first game, where all firms are private and compete à la Cournot-Nash to maximise individual profits. Our first objective is to prove the following claim: 
Proposition 1. The game among profit-maximising firms is a linear state one, and therefore its open-loop Cournot-Nash solution is strongly time consistent.

Proof. The current value Hamiltonian of firm $i$ is:

$$
\mathcal{H}_{i}(t)=\pi_{i}(t)+\lambda_{i}(t) \dot{S}(t)+\mu_{i}(t) \dot{X}(t)
$$

where $\lambda_{i}(t)$ and $\mu_{i}(t)$ are the co-state variables associated with the dynamics of pollution and the natural resource, respectively.

The following system illustrates the set of first order conditions on controls and the associated co-state equations (omitting henceforth the time argument for brevity):

$$
\begin{gathered}
\frac{\partial \mathcal{H}_{i}}{\partial q_{i}}=a-2(1+c) q_{i}-Q_{-i}+b \lambda_{i}-v \mu_{i}=0 \\
-\frac{\partial \mathcal{H}_{i}}{\partial S}=\dot{\lambda}_{i}-\rho \lambda_{i} \Leftrightarrow \dot{\lambda}_{i}=(\rho+\delta) \lambda_{i} \\
-\frac{\partial \mathcal{H}_{i}}{\partial X}=\dot{\mu}_{i}-\rho \mu_{i} \Leftrightarrow \dot{\mu}_{i}=(\rho+\eta) \mu_{i}
\end{gathered}
$$

where $Q_{-i} \equiv \sum_{j \neq i} q_{j}$ is the amount of instantaneous output collectively supplied by all rivals of firm $i$ at any given time. Clearly, (8-10) jointly imply that the optimal output of firm $i$ never depends on the states. The intuitive reason is that firms - being unregulated profit maximising entities are completely uninterested in the amount of pollution and the stock of the resource and consequently behave as if the two-sided tragedy of commons did not exist. From a strictly technical standpoint, one can easily check that

$$
\frac{\partial^{2} \mathcal{H}_{i}}{\partial q_{i} \partial S}=\frac{\partial^{2} \mathcal{H}_{i}}{\partial S^{2}}=0
$$

as well as

$$
\frac{\partial^{2} \mathcal{H}_{i}}{\partial q_{i} \partial X}=\frac{\partial^{2} \mathcal{H}_{i}}{\partial X^{2}}=0
$$

and therefore the game is indeed a linear state one (cf. Dockner et al., 2000, p. 188), yielding a subgame perfect or strongly time consistent Nash equilibrium under the open-loop information structure. 
Accordingly, from (10) one obtains $\mu_{i}=0$ for all $i=1,2,3, \ldots N$ at any time during the game. Then, from (8), one finds

$$
\lambda_{i}=\frac{2(1+c) q_{i}+Q_{-i}-a}{b} .
$$

Then, differentiating w.r.t. time, imposing symmetry across quantities $\left(q_{j}=\right.$ $q_{i}=q$ for all $\left.i, j\right)$ and using (9), the control equation obtains:

$$
\dot{q}=\frac{(\rho+\delta)[q(n+1+2 c)-a]}{2 c+n+1} .
$$

Imposing stationarity, we have $q^{C N}=a /(n+1+2 c)$, which coincides with the solution of the static game. Superscript $C N$ stands for Cournot-Nash. Of course the same solution obtains immediately by observing that the system of co-state equations (9-10) admits the solution $\lambda_{i}=\mu_{i}=0$ for all $i=$ $1,2,3, \ldots N$ at all times, whereby the first order condition (8) indeed delivers $q^{C N}=a /(n+1+2 c)$ throughout the game. ${ }^{6}$

Before proceeding any further, we briefly evaluate the stability properties of the dynamic system (2-4-14), by looking at the associated Jacobian matrix:

$$
J=\left[\begin{array}{ccc}
\frac{\partial \dot{S}}{\partial S} & \frac{\partial \dot{S}}{\partial X} & \frac{\partial \dot{S}}{\partial q} \\
\frac{\partial \dot{X}}{\partial S} & \frac{\partial \dot{X}}{\partial X} & \frac{\partial \dot{X}}{\partial q} \\
\frac{\partial \dot{q}}{\partial S} & \frac{\partial \dot{q}}{\partial X} & \frac{\partial \dot{q}}{\partial q}
\end{array}\right]
$$

that is,

$$
J=\left[\begin{array}{ccc}
-\delta & 0 & b N \\
0 & \eta & -v N \\
0 & 0 & \rho+\delta
\end{array}\right]
$$

whose eigenvalues are

$$
\zeta_{1}=-\delta<0 ; \zeta_{2}=\eta>0 ; \zeta_{3}=\rho+\delta>0 .
$$

\footnotetext{
${ }^{6}$ This also implies that $\lambda_{i}=0$ for all $i$ throughout the game, as is easily verified from (13). Thus, the transversality conditions

$$
\lim _{t \rightarrow \infty} \lambda_{i} S=\lim _{t \rightarrow \infty} \mu_{i} X=0
$$

are trivially satisfied for all $i$.
} 
Accordingly, we can state:

Proposition 2. The Cournot-Nash equilibrium of the open-loop game is a saddle point.

The corresponding amount of pollution and the residual volume of natural resource obtain, respectively, from $\dot{S}=0$ and $\dot{X}=0:^{7}$

$$
\begin{aligned}
S^{C N} & =\frac{n a b}{(2 c+n+1) \delta} ; \\
X^{C N} & =\frac{n a v}{(2 c+n+1) \eta} .
\end{aligned}
$$

From the above expressions we can draw:

Lemma 3. Since

$$
\lim _{n \rightarrow \infty} S^{C N}=\frac{a b}{\delta} ; \lim _{n \rightarrow \infty} X^{C N}=\frac{a v}{\eta},
$$

open access implies positive and finite volumes of resource and pollution at the steady state.

In particular, the second of the above limits reveals that open access does not lead to resource extinction.

The per-firm profits and social welfare in steady state are

$$
\begin{aligned}
\pi^{C N} & =\frac{a^{2}(1+c)}{(2 c+n+1)^{2}} ; \\
S W^{C N} & =\frac{n a \Upsilon}{2(2 c+n+1)^{2} \delta^{2} \eta}
\end{aligned}
$$

with

$$
\begin{aligned}
\Upsilon \equiv & a\left[(n+2(1+c)) \delta^{2}-n b^{2} \gamma\right] \eta \\
& +2 v(2 c+n+1) \delta^{2} .
\end{aligned}
$$

The above expression reveals the following result.

Lemma 4. The condition

$$
\delta>b \sqrt{\frac{n \gamma}{n+2(1+c)}} \equiv \delta_{S W}^{C N}
$$

\footnotetext{
${ }^{7}$ Then, one can also easily show that the feedback equilibrium based upon the linear value function $V_{i}(S, X)=\omega_{1}+\omega_{2} S+\omega_{3} X$ and the corresponding Bellman equation $\rho V_{i}(S, X)=\max _{q_{i}}\left[\pi_{i}+\partial V_{i} / \partial S \cdot d S / d t+\partial V_{i} / \partial X \cdot d S / d t\right]$ is indeed $\left(S^{C N}, X^{C N}, q^{C N}\right)$.
} 
suffices to ensure that the steady state social welfare level of the Cournot game be positive.

That is, if the rate of absorption of pollutants is high enough, then social welfare cannot fall below zero. The critical threshold highlighted in Lemma 4 is clearly increasing in both $b$ and $\gamma$, i.e., the parameters measuring the marginal contribution of industry output to the accumulation of pollution, and the weight of the stock of pollutants in the composition of welfare.

\section{The Bertrand-Nash game}

Here we deal with the price competition case. The Hamiltonian of firm $i$ is defined as in (7), and as usual profit-seeking firms do not internalise the external effects of their strategies. Hence, also in the Bertrand case the openloop equilibrium will be strongly time consistent, and will replicate forever the equilibrium of the static one-shot game. There remains to characterise the equilibrium price behaviour of firms at the Bertrand-Nash equilibrium. To perform this task, we will rely on Dastidar (1995), that has established what follows.

According to Dastidar (1995), if the cost function (common to all firms) features decreasing returns to scale, as is the case in the present model, then the Bertrand-Nash equilibrium is necessarily non-unique. In particular, the pure-strategy Nash equilibrium is characterised by both firms setting the

same price $p^{B N}$, which is bounded by two thresholds, $p^{a v c} \leq p^{B N} \leq p^{u}$. The lower bound $p^{a v c}$ (as the superscript indicates) equals average variable costs, so that at $p^{a v c}$ firms will be indifferent between either producing the output solving

$$
p^{a v c}=a-n q
$$

or producing nothing at all. The upper bound $p^{u}$ (with superscript $u$ standing for undercutting) is the price at which firms are indifferent between choosing price $p^{u}$, and marginally undercutting it in order to capture the entire market demand at $p^{u}$.

The value of $p^{a v c}$ is given by solving (23) w.r.t. $q$ :

$$
c q=a-n q
$$

which delivers $q=a /(c+n)$ and then substituting the latter into the demand function to obtain:

$$
p^{a v c}=\frac{a c}{c+n} .
$$


The upper bound to the equilibrium price, $p^{u}$, obtains by imposing indifference between the symmetric oligopoly profits:

$$
\pi_{O}=(a-n q) q-c q^{2}
$$

and the monopoly profits generated by undercutting:

$$
\pi_{M}=n\left[(a-n q) q-c n q^{2}\right] .
$$

Imposing $\pi_{O}=\pi_{M}$ we obtain the following:

$$
q=\frac{a}{c(n+1)+n}
$$

which can be plugged into the demand function to identify

$$
p^{u}=\frac{a(n+1) c}{c(n+1)+n} .
$$

As a matter of curiosity, one can equate the inverse demand function to marginal cost, then solve for $q$ and finally substitute the resulting output back into the demand function, to obtain marginal cost pricing:

$$
p^{m c}=\frac{2 a c}{2 c+n},
$$

where, obviously, superscript mc stands for marginal cost pricing.

The continuum of Nash equilibria can be represented by the following expression:

$$
p^{B N}=\frac{a c}{c+n-\alpha} .
$$

Parameter $\alpha$ represents the relative intensity of price competition between firms. Note that, when $\alpha=0$, the equilibrium price $p^{B N}$ equals average variable cost; $\alpha=1$ corresponds to the case in which price equals marginal cost, while at $\alpha=4 / 3$ the price attains the highest level above which undercutting takes place. As a consequence, we impose the restriction $\alpha \in[0,4 / 3]$. Using (31), the individual output and profits write, respectively, as follows:

$$
\begin{gathered}
q^{B N}=\frac{a(n-\alpha)}{n(c+n-\alpha)} \\
\pi^{B N}=\frac{a^{2} c(n-\alpha) \alpha}{n^{2}(c+n-\alpha)^{2}} .
\end{gathered}
$$


Using (32), we can solve $\dot{S}=0$ and $\dot{X}=0$ to obtain the steady state levels of pollution and the natural resource:

$$
\begin{aligned}
S^{B N} & =\frac{a(n-\alpha) b}{(c+n-\alpha) \delta} \\
X^{B N} & =\frac{a(n-\alpha) v}{(c+n-\alpha) \eta} .
\end{aligned}
$$

It is worth noting that the limits of $S^{B N}$ and $X^{B N}$ coincide with those of the corresponding magnitudes generated by Cournot behaviour. ${ }^{8}$

The associated level of social welfare is:

$$
S W^{C N}=\frac{a(n-\alpha) \Phi}{2 n(c+n-\alpha)^{2} \delta^{2} \eta}
$$

with

$$
\begin{gathered}
\Phi \equiv 2 n v(c+n-\alpha) \delta^{2}+ \\
\alpha\left[n(n-\alpha)\left(\delta^{2}-b^{2} \gamma\right)+2 c \alpha \delta^{2}\right] \eta .
\end{gathered}
$$

The above expression immediately shows the following.

Lemma 5. The condition

$$
\delta>b \sqrt{\gamma} \equiv \delta_{S W}^{B N}
$$

suffices to ensure that the steady state social welfare level of the Bertrand game be positive.

Since

$$
\frac{n}{n+2(1+c)}<1
$$

for all $n \geq 2$ and $c>0$, the threshold emerging from the Cournot game is lower than the one characterising the Bertrand setting.

\section{The second-best Industry structure}

Here we focus on the issue of the optimal number of firms in the commons. We set out by checking that the industry structure maximising industry

\footnotetext{
${ }^{8}$ Of course the steady state levels of resource and pollution do not coincide in general across the two settings. A general appraisal of Cournot vs Bertrand is given in section 5 .
} 
profits in the steady state of the Cournot model solves

$$
\frac{\partial\left(n \pi^{C N}\right)}{\partial n}=\frac{a^{2}(1+c)(2 c-n+1)}{(2 c+n+1)^{3}}=0
$$

in $n_{\Pi}^{C N}=2 c+1$, so that $n_{\Pi}^{C N} \geq 2$ for all $c \geq 1 / 2$, otherwise $n_{\Pi}^{C N}=1$ and industry profits are maximised in monopoly. Suppose indeed $c \geq 1 / 2$. If so, we can assess the social (in)efficiency of the privately optimal number of firms $n_{\Pi}^{C N}$ by substituting $n_{\Pi}^{C N}=1+2 c$ into the first derivative of $S W^{C N}$ w.r.t. $n$, obtaining:

$$
\left.\frac{\partial S W^{C N}}{\partial n}\right|_{n_{\Pi}^{C N}} \propto(a \eta+2 v) \delta^{2}-a b^{2} \gamma \eta \gtrless 0
$$

for all

$$
\delta \gtrless b \sqrt{\frac{a \gamma \eta}{a \eta+2 v}} \equiv \delta_{n}^{C N}
$$

and the r.h.s. of (41) may be evaluated against the threshold $\delta_{S W}^{C N}$ appearing in Lemma 4 at $n=n_{\Pi}^{C N}=1+2 c$ :

$$
\begin{gathered}
\sqrt{\frac{a \eta}{a \eta+2 v}} \gtrless \sqrt{\frac{2 c+1}{3+4 c}} \\
\Leftrightarrow a \gtrless \frac{v(1+2 c)}{(1+c) \eta} .
\end{gathered}
$$

Therefore we can formulate:

Proposition 6. Take $a>v(1+2 c) /[(1+c) \eta]$ and $\delta \geq \delta_{S W}^{C N}$, so that $S W^{C N} \geq 0$. In this range, $\delta_{n}^{C N}>\delta_{S W}^{C N}$ and

$$
\begin{aligned}
& \left.\frac{\partial S W^{C N}}{\partial n}\right|_{n_{\Pi}^{C N}}<0 \forall \delta \in\left[\delta_{S W}^{C N}, \delta_{n}^{C N}\right) ; \\
& \left.\frac{\partial S W^{C N}}{\partial n}\right|_{n_{\Pi}^{C N}} \geq 0 \forall \delta \geq \delta_{n}^{C N} .
\end{aligned}
$$

In the alternative range $a<v(1+2 c) /[(1+c) \eta]$, we have $\delta_{n}^{C N}<\delta_{S W}^{C N}$ and consequently

$$
\left.\frac{\partial S W^{C N}}{\partial n}\right|_{n_{\Pi}^{C N}} \geq 0 \forall \delta \geq \delta_{S W}^{C N} .
$$


Now we turn our attention to the Bertrand case. Here, the number of firms that maximises industry profits $n \pi^{B N}$ solves

$$
3 n^{3}-\alpha^{2}+2 \alpha(2 n+c)-\alpha\left(6 n^{2}+c(4 n+c)\right)=0
$$

delivering ${ }^{9}$

$$
n_{\Pi}^{B N}=\frac{3 \alpha+\sqrt{\alpha(\alpha+8 c)}}{4} \geq 2
$$

for all $c \geq(4-\alpha)(2-\alpha) / \alpha>1 / 2$ for all $\alpha \in[0,4 / 3]$. Under this condition (that excludes the trivial monopoly case and ensures $n \geq 2$ irrespective of the market variable being set), we can substitute $n_{\Pi}^{B N}$ into the derivative of $S W^{B N}$ w.r.t. $n$, finding

$$
\left.\frac{\partial S W^{B N}}{\partial n}\right|_{n_{\Pi}^{B N}} \propto a \alpha \eta\left(\delta^{2}-b^{2} \gamma\right) \Omega+\delta^{2} v \Gamma,
$$

with

$$
\begin{gathered}
\Omega \equiv 10 c+(2 c+1) \sqrt{\alpha(\alpha+8 c)}-\alpha>0 ; \\
\Gamma \equiv 4(5 \alpha+2 c) c-\alpha^{2}+(\alpha+8 c) \sqrt{\alpha(\alpha+8 c)}>0 .
\end{gathered}
$$

Since Lemma 5 establishes that $\delta>\delta_{S W}^{B N}=b \sqrt{\gamma}$ in order for $S W^{B N}>0$, we can state:

Proposition 7. Take $c \geq(4-\alpha)(2-\alpha) / \alpha>1 / 2$, so that $n_{\Pi}^{B N} \geq 2$. $\partial S W^{B N} /\left.\partial n\right|_{n_{\Pi}^{B N}}>0$ for all $\delta>\delta_{S W}^{B N}$.

We may sum up this discussion in the following terms. Provided $\delta$ is large enough to ensure that social welfare is positive at the steady state of both games, Propositions 6 and 7 jointly convey a message telling that, while under quantity-setting behaviour the privately optimal number of firms may be higher or lower than the socially optimal one, depending on market size and the environment's efficiency in absorbing pollution, under price-setting behaviour we have the unambiguous result that a benevolent planner would like to enlarge the population of firms as compared to the privately optimal industry structure. An explanation of this result is sketched in the next section.

\footnotetext{
${ }^{9}$ The other solution to $(43)$ can be excluded as it is negative for all $\alpha \in[0,4 / 3]$.
} 


\section{Prices vs quantities}

The comparative assessment of the two settings can be carried out along several dimensions, the first being the relative size of $n_{\Pi}^{C N}$ vs $n_{\Pi}^{B N}$. The expression $n_{\Pi}^{B N}-n_{\Pi}^{C N}$ is concave in $\alpha$, and $n_{\Pi}^{B N}=n_{\Pi}^{C N}$ in

$$
c=\frac{7 \alpha-8 \pm \sqrt{\alpha(17 \alpha-16)}}{16}
$$

with $c_{-}<0$ for all $\alpha \in[16 / 17,4 / 3]$ and $c_{+} \geq 0$ for all $\alpha \in[1,4 / 3]$. Yet, $c_{+}<$ $(4-\alpha)(2-\alpha) / \alpha$ for all admissible values of $\alpha$, and consequently $n_{\Pi}^{B N}<$ $n_{\Pi}^{C N}$ except in the trivial case where both models only accomodate monopoly. The fact that Cournot behaviour allows for a larger population of firms in correspondence of maximum industry profits is definitely behind the result emerged from Propositions 6-7 above.

Consider now output levels, given a generic number of firms. The difference:

$$
q^{B N}-q^{C N} \propto n-\alpha+(n-2 \alpha) c
$$

is surely positive (i) for any $n \geq 2$, if $\alpha \in[0,1]$; (ii) for any $\alpha \in[0,4 / 3]$, if $n \geq 3$. Taking into account the integer problem and the constraint $c \geq$ $(4-\alpha)(2-\alpha) / \alpha$ inherited from Proposition 7, the r.h.s. of (49) changes sign in the region identified by $n=2$ and $\alpha \in[1,(9-\sqrt{17}) / 4)$, in which

$$
q^{B N} \gtrless q^{C N} \text { for all } c \lessgtr \frac{n-\alpha}{2 \alpha-n}=\frac{2-\alpha}{2(\alpha-1)} \text {. }
$$

If instead $\alpha \in((9-\sqrt{17}) / 4,4 / 3)$, we have

$$
\frac{2-\alpha}{2(\alpha-1)}>\frac{(4-\alpha)(2-\alpha)}{\alpha}
$$

and therefore $q^{C N}>q^{B N}$. Exactly the same conclusions apply for aggregate industry output and therefore also for the steady state levels of pollution and the natural resource, as the latter are, by the assumptions of the model, linear in the industry output. Hence, we may conclude that, taking $n \geq$ 2 as given (with the exception of the particular and circumscribed case identified in (50)), pollution is lower under Bertrand competition than under Cournot competition, due to the output restriction observed under pricesetting behaviour, which is generated by the presence of decreasing returns to 
scale. Exactly the opposite would intuitively apply if the marginal cost were constant, as in such a case the Bertrand-Nash equilibrium would coincide with perfect competition and consequently industry output would be higher than the Cournot-Nash one, with obvious consequences on industry profits. However, by the same token, consumer surplus and the steady state volume of natural resource are both higher under Cournot competition than under Bertrand competition.

A different exercise can be envisaged to compare the two industry output given their respective optimal industry structures $n_{\Pi}^{C N}$ and $n_{\Pi}^{B N}$, to evaluate the difference

$$
\begin{gathered}
\Delta Q=\left.Q^{B N}\right|_{n_{\Pi}^{B N}}-\left.Q^{C N}\right|_{n_{\Pi}^{C N}}= \\
\frac{\alpha+2 c-\sqrt{\alpha(\alpha+8 c)}}{4(\alpha-c)},
\end{gathered}
$$

whose numerator is positive for all $c>\alpha$ (and conversely), so that $\Delta Q<0$ everywhere. ${ }^{10}$ For the aforementioned reasons, this has in principle ambiguous consequences on welfare. Hence we must evaluate

$$
\left.\Delta S W \equiv W^{B N}\right|_{n_{\Pi}^{B N}}-\left.W^{C N}\right|_{n_{\Pi}^{C N}}
$$

In the special case $\alpha=0$ (i.e., under average cost pricing under Bertrand behaviour), we have:

$$
\Delta S W \propto-\left[a \eta\left(\delta^{2}-b^{2} \gamma\right)+2 v \delta^{2}\right](1+2 c)-2 c v \delta^{2}<0
$$

for any $\delta>b \sqrt{\gamma}$. For any $\alpha \in(0,4 / 3]$, we have instead: ${ }^{11}$

$$
\Delta S W=\gamma \Lambda(a, b, c, v, \alpha, \delta, \eta)-\Xi(a, b, c, v, \alpha, \delta, \eta)
$$

with $\Lambda(\cdot), \Xi(\cdot)>0$, so that $\Delta S W>0$ for all $\gamma>\Xi(\cdot) / \Lambda(\cdot)$. Accordingly, we may state:

Proposition 8. In correspondence of the optimal industry structure $n_{\Pi}^{K N}$, $K=B, N$, the steady state social welfare level is higher under Cournot behaviour for all admissible levels of $\gamma$, if average cost pricing prevails in the Bertrand game. Otherwise, for positive values of $\alpha$, steady state social welfare is higher under Cournot competition if $\gamma$ is high enough.

\footnotetext{
${ }^{10}$ The possibility for Cournot to accomodate more firms (and therefore deliver a higher output) than Bertrand has been highlighted in a static game by Cellini, Lambertini and Ottaviano (2004).

${ }^{11}$ We omit the expressions $\Lambda(\cdot)$ and $\Xi(\cdot)$ for brevity. These can be reconstructed from the definition of $\Delta S W$ in $(53)$.
} 


\section{Conclusions}

We have analysed a differential oligopoly game in which environmental externalities and the exploitation of natural resources combine in a single framework. Considering prices or quantities alternatively as the firms' strategic instruments, we have assessed the privately optimal number of firms against the socially optimal one, showing the emergence of an ambiguous conclusion in the Cournot setup. Conversely, under Bertrand behaviour the privately optimal degree of concentration is definitely too large from the social standpoint. Relatedly, taking as a benchmark the privately optimal industry structure, we have shown that the relative size of welfare levels at the steady states of the two models depends on the capability of the environment to absorb polluting emissions.

The foregoing analysis has been carried out assuming any form of regulation away. The study of the interplay between environmental policy, firms' strategic behaviour and the (in)efficiency of the resulting industry structure in the commons is left for future research. 


\section{REFERENCES}

Anderson, D. (2010). Environmental Economics and Natural Resource Management. Third Edition. London, Routledge.

Benchekroun, H. and N.V. Long (1998). Efficiency inducing taxation for polluting oligopolists. Journal of Public Economics, 70, 325-342.

Benchekroun, H. and N.V. Long (2002). On the multiplicity of efficiencyinducing tax rules. Economics Letters, 76, 331-336.

Cellini, R., L. Lambertini and G. Ottaviano (2004). Welfare in a differentiated oligopoly with free entry: a cautionary note. Research in Economics, 58, 125-133.

Cornes, R. and T. Sandler (1983). On commons and tragedies. American Economic Review, 73, 787-792.

Cornes, R., C.F. Mason and T. Sandler (1986). The commons and the optimal number of firms. Quarterly Journal of Economics, 101, 641646.

Dasgupta, P.S. and G.M. Heal (1979). Economic Theory and Exhaustible Resources. Cambridge, Cambridge University Press.

Dastidar, G.S. (1995). On the existence of pure strategy Bertrand equilibrium. Economic Theory, 5, 9-32.

Dockner, E., S. Jørgensen, N.V. Long and G. Sorger (2000). Differential Games in Economics and Management Science. Cambridge, Cambridge University Press.

Fujiwara, K. (2009). Environmental policies in a differentiated oligopoly revisited. Resource and Energy Economics, 31, 239-247.

Gordon, H.S. (1954). The economic theory of a common-property resource: the fishery. Journal of Political Economy, 62, 124-142.

Hardin, G. (1968). The tragedy of the commons. Science, 162, 1243-1248. 
Karp, L. (1992). Social welfare in a common property oligopoly. International Economic Review, 33, 353-372.

Karp, L. and J. Livernois (1992). On efficiency-inducing taxation for a nonrenewable resource monopolist. Journal of Public Economics, 49, 219-239.

Katsoulacos, Y. and A. Xepapadeas (1995). Environmental policy under oligopoly with endogenous market structure. Scandinavian Journal of Economics, 97, 411-420.

Kemp, M.C. and N.V. Long (1980). Resource extraction under conditions of common access. In M.C. Kemp and N.V. Long (eds), Exhaustible Resources, Optimality and Trade, Amsterdam, North-Holland.

Mankiw, N.G. and M.D. Whinston (1990). Free entry and social inefficiency. RAND Journal of Economics, 17, 48-58.

Mason, C.F. and S. Polasky (1997). The optimal number of firms in the commons: a dynamic approach. Canadian Journal of Economics, 30, 1143-1160.

McMillan, J. and H.W. Sinn (1984), "Oligopolistic Extraction of a CommonProperty Resource: Dynamic Equilibria", in M.C. Kemp and N.V. Long (eds), Essays in the Economics of Exhaustible Resources, Amsterdam, North-Holland.

Novshek, W. (1980). Cournot equilibrium with free entry. Review of Economic Studies, 47, 473-486.

Pearce, D.W. and R.K. Turner (1989). Economics of Natural Resources and the Environment. Hemel Hempstead, Harvester-Wheatsheaf.

Sinn, H.W. (1984). Common property resources, storage facilities and ownership structures: a Cournot model of the oil market", Economica, 51, $235-252$.

Sunnevåg, K.J. (2003). Auction design for the allocation of emission permits in the presence of market power. Resource and Energy Economics, 26, $385-400$. 
Suzumura, K. and K. Kiyono (1987). Entry barriers and economic welfare. Review of Economic Studies, 54, 157-167.

Tisdell, C. (2009). Resource and Environmental Economics. Modern Issues and Applications. Singapore, World Scientific.

von der Fehr, N.-H. (1993). Tradeable emission rights and strategic interaction. Environmental and Resource Economics, 3, 129-151. 


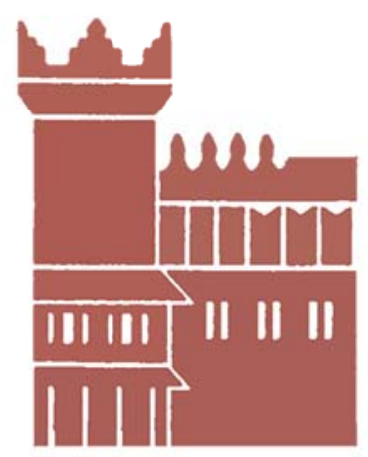

Alma Mater Studiorum - Università di Bologna DEPARTMENT OF ECONOMICS

Strada Maggiore 45

40125 Bologna - Italy

Tel. +39051 2092604

Fax +390512092664

http://www.dse.unibo.it 